\title{
Incorporación de harina de pimentón en la alimentación de ponedoras y capacitación de productores avícolas asociados a AVIMETA
}

\section{Adding paprika fluor in feeding of commercial egg laying poultry producers and training associated AVIMETA}

\author{
Barreto S. Yenny A. ${ }^{1}$, Ávila V. José Y. ${ }^{1}$ y Lozada M. Hernando² \\ ${ }^{1}$ Licenciados en Producción Agropecuaria, \\ ${ }^{2}$ MVZ. Docente Universidad de los Llanos \\ hdolozada@yahoo.com.ar
}

Recibido 2 de Junio 2012, Aprobado 3 de Octubre 2012

\section{RESUMEN}

Dos estudiantes del programa de Licenciatura en Producción Agropecuaria de la Universidad de los Llanos, desarrollaron un proyecto para utilizar harina de pimentón (Capsicum annuum), como fuente de carotenoides para mezclarla con el alimento concentrado para gallinas ponedoras y observar cómo contribuyen con la pigmentación de las yemas de huevos producidos por ponedoras mudadas de ochenta y dos (82) semanas de vida, en una granja avícola (Agropecuaria río Ocoa) localizada sobre el kilómetro siete (7) vía Villavicencio - Puerto López (Meta). Para ejecutar el proyecto, se tomaron al azar ciento veinte (120) aves y se dividieron en dos grupos. Un grupo testigo conformado por veinte (20) ponedoras a las que se les suministró el alimento concentrado comercial y el manejo tradicional. Un grupo experimental de cien (100) ponedoras dividido en cinco (5) subgrupos de veinte (20) ponedoras cada uno, a las que se les suministró cantidades crecientes de harina de pimentón en proporciones del 0.5, 1.0, 1.5, 2.0 y $2,5 \%$ respectivamente. Por otro lado, el trabajo tuvo un componente pedagógico dirigido al personal operativo y directivos de la asociación de avicultores del Meta "AVIMETA", que manejan ocho (8) granjas productoras de huevo comercial para consumo, en el departamento del Meta. Para capacitar el personal operativo, se ejecutó un proceso que incluyó tres (3) estrategias formativas: Una demostración de campo, un manual didáctico y tres seminarios. (Un seminario para cada zona donde trabajan los operarios). Adicionalmente generó el espacio apropiado para 
que los trabajadores de la granja avícola "Agropecuaria río Ocoa", las directivas de AVIMETA y otros avicultores, pudieran observar de primera mano, los resultados que se obtuvieron en cada una de las tres (3) semanas que duró ese componente del trabajo.

Palabras clave: Ponedora, harina de pimentón, carotenoides.

\begin{abstract}
Two students of Agricultural Production, University of the Llanos, developed a project to use flour pepper (Capsicum annuum), as a source of carotenoids to mix with the concentrate for laying hens and watch these carotenoids contribute with the pigmentation of egg yolks produced by hens molted eighty-two (82) weeks, at a poultry farm (Agricultural Ocoa River) located at kilometer seven (7) track Villavicencio - Puerto López (Meta). To run the project, were randomly hundred twenty (120) layers and divided them into two groups. A control group consisting of twenty (20) to which hens were fed the commercial supplement and traditional management. An experimental group of one hundred (100) layers divided into five (5) sub-groups of twenty (20) layers each, which were given increasing amounts paprika flour in proportions of $0.5,1.0,1.5,2.0$ and $2.5 \%$ respectively. Furthermore, the work had an educational component for staff and operational managers poultry association Meta "AVIMETA" handlers eight (8) commercial eggproducing farms for consumption, in the department of Meta. To train operational staff, ran a process that included three (3) training strategies: A field demonstration, a training manual and three seminars. (A seminar for each work area where workers). Additionally, generated the appropriate space for the workers of the poultry farm "Agricultural Ocoa River" AVIMENTA policies and other poultry, could observe first hand, the results obtained in each of the three (3) weeks of that component of the work.
\end{abstract}

Keywords: Laying, paprika flour, carotenoids. 


\section{INTRODUCCIÓN}

En Colombia, la producción avícola presentó una fase inicial de crecimiento significativo, en la década 1990-2000, pero ahora está por debajo de los promedios y solo a nivel de los países andinos, la avicultura colombiana muestra una mayor participación en el volumen de producción (Fenavi, 2007). En el año 2006 la producción llego alrededor de 6.800 millones de unidades (67\% huevo rojo, 33\% huevo blanco) en granjas con 1.000 hasta 500.000 ponedoras. La dinámica mostró, para el año 2004, un crecimiento del $10,22 \%$ y se preveía un decrecimiento del 4,56\% para el 2006. La participación regional fue la siguiente: Zona Central (Cundinamarca, Meta, Tolima y Huila) 35,8\%; Santanderes 24,7\%; Valle del Cauca 21,5\%; Antioquia 9\%; Costa Atlántica 5\%; el Eje Cafetero con $4,3 \%$ y el Oriente del país con $2 \%$. En términos del consumo de huevo, en los últimos años ha estado entre 7,6 Kilos/habitante/año (según estadísticas nacionales) y 8,1 kilos, según la FAO esto resulta cercano a los promedios mundiales, pero no por ello satisfactorio (Mora, 2007).

En el departamento del Meta, la producción de huevo es una actividad emprendida por avicultores que generalmente tienen una participación integral en el mercado, porque la mayoría $(84,6 \%$ ), se dedica a la ceba de pollo (Gobernación del Meta, 2006). Gran parte de la producción se comercializa a través de los almacenes de cadena, que son exigentes y su personal de recepción se toma el trabajo de revisar cada una de las unidades suministradas, para rechazar aquellas que tienen alteraciones en la cáscara (fisuras-porosidades-deformidades) y pigmentaciones irregulares.

En un proceso posterior a la primera venta, la pigmentación de la yema juega un papel fundamental, pues los consumidores domésticos (no los industriales), para comprar huevos se guían por la coloración, ya que mientras más pigmentada sea la yema, el huevo se considera de mejor calidad. Adicionalmente, en el mercado siempre ha habido gran interés por la producción de los llamados "huevos de campo" (Gobernación del Meta, 2006), que se caracterizan por la alta pigmentación de la yema y el color marrón de la cáscara (Figura 1), que 
actualmente tienen gran demanda en el mercado de Villavicencio y Bogotá. Sin embargo, la nutrición de las ponedoras se continúa haciendo mediante el suministro de concentrado tradicional como alimento completo, cuya composición varía de conformidad con la fase de desarrollo del animal; es así como se encuentran algunas formulaciones específicas para pollitas (semanas 0-6), otras para el levante (semanas 7-14), otras para la pre postura (semanas 15-19) y normalmente otras para la fase definitiva de postura (semanas 20-52).
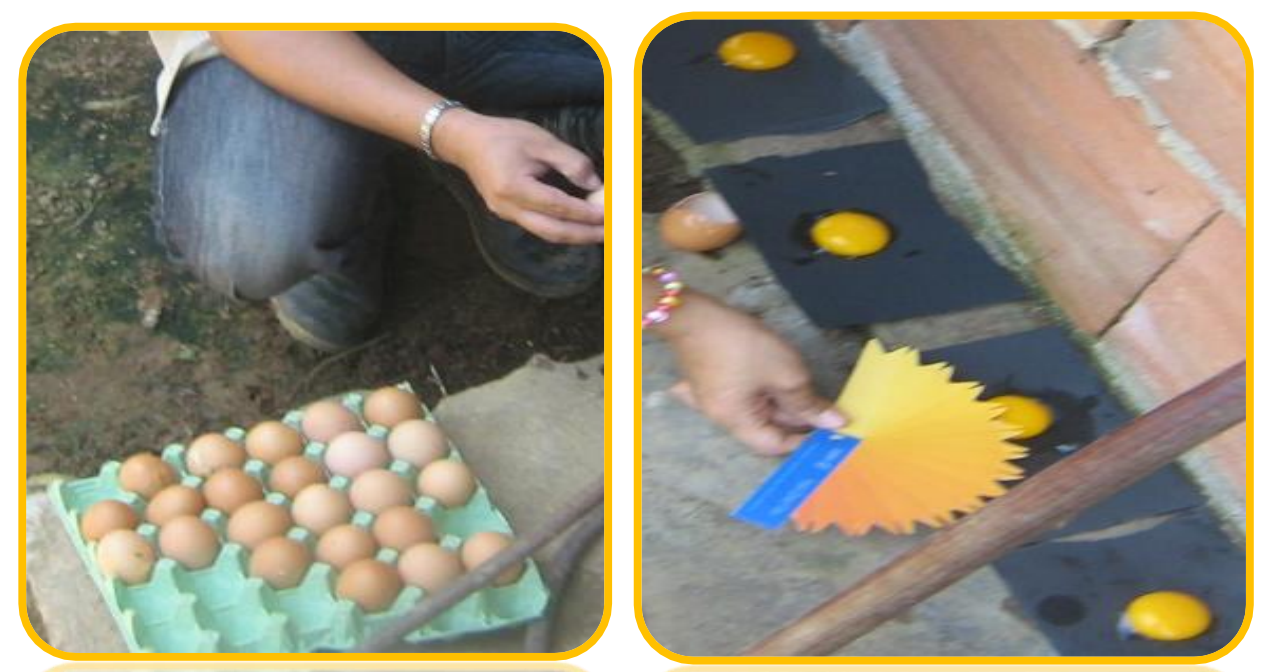

Figura 1. Recolección de datos de los huevos de campo, observando su coloración

Es de común conocimiento que los alimentos concentrados para aves de postura, incorporan en sus ingredientes una costosa fuente sintética de carotenos conocida industrialmente como Carofil (Ester etílico del ácido apocarotenoico), que determina la coloración de la yema de los huevos para consumo. Este factor, sumado a que por sus particularidades fisiológicas las aves no sintetizan carotenoides, determina que la intensidad del color de la yema dependa exclusivamente del aporte de carotenos presentes en la dieta proveída, con el agravante de que a medida que la ponedora envejece, va perdiendo gran parte de la capacidad para trasladar esos pigmentos (Rivera, 2006). 


\section{LAS BONDADES DEL PIMENTÓN}

En el campo agrícola, Colombia cuenta con una gran variedad de cultivos de especies hortícolas, entre las cuales merece especial mención el pimentón, hortaliza que adquiere importancia por sus múltiples usos a nivel industrial y en el mercado de productos frescos. Del pimentón (Capsicum annuum L.) se obtiene una de las más altas concentraciones de carotenoides derivados de fuentes vegetales. Además, son de interés sus propiedades nutricionales, farmacológicas y colorantes (Jaren y Minguez, 1999). La planta del pimentón es una piperácea originaria de México, Bolivia y Perú, donde además del Capsicum annuum se cultivaban al menos otras cuatro especies. El principal componente del pigmento es al agua, seguido de los hidratos de carbono, lo que hace que sea una hortaliza con un bajo aporte calórico. Es una buena fuente de fibra y, al igual que el resto de verduras, su contenido proteico es muy bajo y apenas aporta grasas. En cuanto a su contenido en vitaminas, los pimentones son muy ricos en vitamina $\mathrm{C}$, sobre todo los de color rojo. Son buena fuente de carotenos, entre los que se encuentra la capsantina, pigmento que aporta el característico color rojo

La planta puede ser anual, bianual, o vivir varios años. Posee un tallo lleno de ramas y ésta alcanza los 0,5-1,5 metros. Sus flores son blancas y los frutos pueden variar de color dependiendo del grado de madurez en el que se encuentren. Mientras que la especie puede tolerar la mayoría de los climas, es especialmente productiva en zonas cálidas y climas secos. Se cultiva en todo el mundo, y generalmente se suele comercializar en diferentes colores: verde, rojo y amarillo. Dentro de esta especie se puede encontrar numerosas variedades, generadas por diferencias en el clima, las condiciones del suelo y otros aspectos que influyen en su cultivo.

Los carotenos son un grupo de carotenoides los cuales son una clase de pigmentos terpenoides con 40 átomos de carbono derivados biosintéticamente a partir de dos unidades de geranil-geranil-pirofosfato, en su mayoría son solubles en solventes apolares y de coloraciones que oscilan entre el amarillo (por ejemplo, el ß-caroteno) y el rojo (por ejemplo, el licopeno). Otorgan el color a muchas frutas 
y verduras amarillas, naranjas y rojas. Incluso se ha hallado que los carotenoides confieren brillantes colores a los animales, por ejemplo, los flamencos y los crustáceos deben su color a los carotenoides que previamente obtienen con su dieta. Las yemas de huevo son amarillas por la presencia de carotenoides, que además protegen a las grasas insaturadas que contiene (Olson, 1999).

Los carotenoides se encuentran principalmente en partes aéreas de las plantas, especialmente en hojas, tallos y flores, en frutos (tomate, pimentón, y otras plantas) y en menor proporción en raíces (zanahoria). Los carotenoides junto con las clorofilas, son los pigmentos vegetales más distribuidos (Martinez, 2003). Los carotenoides son sustancias muy sensibles a la luz, el oxígeno, el calor y la humedad, por tanto, es imperativo que sean incorporados en la elaboración del concentrado. La pérdida de carotenoides durante la fabricación y almacenamiento del alimento balanceado resultan en pigmentaciones no uniformes de la yema, o la piel del pollo, lo que da al consumidor la impresión de calidad variable de estos productos. La pigmentación de tejidos como el huevo y la piel, es un proceso natural. Las aves no pueden sintetizar carotenoides, pero tienen la capacidad de absorberlos de la dieta y depositarlos en la yema, la piel, y el tejido adiposo (Cuevas, 2009).

\section{CAROTENOS UTILIZADOS EN ALIMENTOS PARA AVES}

La materia prima utilizada para producir los alimentos de las aves no contiene cantidades suficientes de carotenoides (Rodríguez, 1999) para lograr el color agradable que demandan los consumidores del producto final. Por lo tanto, los carotenoides tienen que ser adicionados al alimento de las aves. El Carofil -o Carophyll, en inglés- es el nombre con el que se denomina el colorante que se incorpora en los alimentos formulados para la fase de pre-postura y postura de ponedoras. Este colorante se encuentra en dos variedades: Rojo y amarillo; el primero es un producto diseñado para un uso muy eficiente en las plantas de producción de alimento concentrado, tiene una presentación en microesferas, las cuales están distribuidas en una matriz de gelatina y carbohidrato, protegidas con una capa de almidón. Tienen el tamaño y la densidad óptimos para fluir libremente 
en la mezcla de ingredientes que conforman el concentrado, pues contiene aproximadamente 100.000 microesferas recubiertas/gramo, oscilando su diámetro entre 0,15 a 0,4 milímetros y un peso específico de 0,7 gramos $/ \mathrm{cm}^{3}$. La forma y la uniformidad de su tamaño aseguran que la sustancia activa -cantaxantina- se distribuya homogéneamente en el alimento concentrado. Mientras que el segundo es un producto comercial del éster etílico del ácido apocarotenoico, cuya concentración es del $10 \%$ y se presenta en forma de polvo granulado. La estandarizada manufactura de Carofil amarillo ha sido diseñada para asegurar un producto consistente con alta estabilidad, extraordinarias propiedades de mezclado y excelente absorción, lo que asegura una mayor uniformidad de la pigmentación de la yema y piel del pollo.

La calidad nutricional de los huevos de gallina no tiene nada que ver con el tamaño, con el color de la cáscara (característica genética) ni con la intensidad de la pigmentación de la yema, pues el pigmento no posee valor nutritivo (Meléndez et al., 2007). En otros tiempos, una yema de color amarillo intenso indicaba que la gallina era alimentada de modo natural y saludable, a base de maíz y otros vegetales frescos que contienen este pigmento natural. Esto, sin embargo, ya no es habitual, pues en las granjas avícolas se les administra a las gallinas el Carofil y otros pigmentos, incorporados en el concentrado.

\section{INCORPORACIÓN DE HARINA DE PIMENTÓN EN LA ALIMENTACIÓN DE PONEDORAS}

El concentrado para ponedoras se suministra como alimento completo desde el comienzo de la producción hasta la semana 72 de vida $(77 \%$ a $80 \%$ de producción). Aves por encima de las 60 semanas de vida tienden a disminuir el consumo de alimento, razón por la cual se debe estimular el consumo.

Dosificación: En ponedoras comerciales debe ser controlada la cantidad de alimento, de acuerdo con la línea, el clima y el peso del ave. El consumo normal diario está entre 115 y 120 gramos/ave. Estas cantidades dependen de la raza, del clima y del tipo de alojamiento. Si se presentan grandes limitaciones en el 
consumo de alimento, esto traerá como consecuencia, aves de bajo peso y falta de persistencia en la postura. Un excesivo consumo de alimento, trae como consecuencia el engrasado de las aves, postura intra-abdominal, prolapsos y alta mortalidad.

Composición: Aunque la composición del alimento puede variar levemente entre las diferentes marcas comerciales, en general debe tener la siguiente composición

Tabla 3. Composición general alimento para ponedoras

\begin{tabular}{cc}
\hline Nutriente & Contenido $(\%)$ \\
\hline Proteína mínimo & 17.0 \\
Grasa mínimo & 2.5 \\
Fibra máximo & 6.0 \\
Cenizas máximo & 15.0 \\
Humedad máximo & 13.0 \\
Calcio mínimo & 3.0 \\
Fósforo mínimo & 0.7 \\
\hline
\end{tabular}

Fuente: Fábrica de alimentos SOLLA. Ponedoras uno (2009).

La harina de pimentón se incorpora al alimento concentrado para las aves, con el propósito de proveerles una fuente adicional de carotenoides, que les permita poner huevos con yema pigmentada. Eso facilita su comercialización porque la gente los prefiere así, en lugar de comprar huevos con yema despigmentada. Sin embargo, la incorporación de la harina de pimentón no se puede hacer "al tanteo", se requiere un procedimiento para garantizar que los carotenoides estén en la cantidad suficiente y en la forma adecuada para que sean absorbidos por las ponedoras.

El procedimiento incluye la selección de los pimentones que se van a utilizar, el escaldado, troceado, secado, molida y la mezclada.

- Selección. Se debe seleccionar pimentones frescos, totalmente rojos y sin evidencias de enfermedades.

- Escaldado. El pimentón fresco se escalda durante cinco minutos a $60^{\circ} \mathrm{C}$. Este procedimiento inactiva la descomposición oxidativa de los carotenoides, además mejora color, textura, sabor, y provoca un 
reblandecimiento de los tejidos lo que permite disminuir el tiempo de secado y de extracción (Owen, 2000).

- Troceado. Sobre una superficie para picar el pimentón escaldado, al que se le retira la semilla y se le corta en tiras de aproximadamente $0,5 \times 3,0$ centímetros. Se debe evitar la exposición de las tiras a la luz solar o artificial directa, por lo cual hay que hacerlo de manera rápida.

- Secado. A las tiras de pimentón se les coloca un ventilador, durante un tiempo de 8 horas, a una temperatura media de $24^{\circ} \mathrm{C}$; la velocidad del aire para secar las tiras se regula en el nivel más bajo, buscando acercarse a $0.2 \mathrm{~m}^{3} /$ hora. Adicionalmente las ventanas se cubren con plásticos negros, para proteger el material de la luz.

- Molido. Para obtener un tamaño de partícula fina, se puede utilizar un molino casero. El proceso tiene que ser muy rápido para mantener las condiciones de tonalidad y gama de color del pimentón.

- Incorporado. En esta acción la harina de pimentón se adiciona en el alimento de las ponedoras, en las cantidades precisas para producir el efecto deseado, sin afectar la ingestión del alimento ni el porcentaje de postura (Figura 2). Se recomienda pesar una cantidad de harina de pimentón equivalente al dos (2\%) por ciento de la ración e incorporarla con la cantidad de concentrado que se les suministra diariamente a las ponedoras.

Como puede apreciarse, la cantidad de harina requerida es mínima, comparada con lo que come la ponedora, así que es necesario mezclar muy bien, para que la distribución sea homogénea y la ponedora consuma los carotenoides en la cantidad apropiada. Expresado lo anterior de manera aritmética, sería de la siguiente forma: si cada ponedora consume normalmente $121 \mathrm{~g} /$ día, se requiere adicionar una cantidad de harina de pimentón equivalente al $2 \%$, es decir 2.42 gramos. Para no manejar cantidades tan pequeñas, podemos tomar como regla general lo siguiente: para cien (100) gallinas, se requiere mezclar 12,1 kilos de 
concentrado/día, con 242 gramos de harina de pimentón. Por ningún motivo se debe mezclar harina húmeda con el concentrado, porque lo estropea y predispone a que las ponedoras se enfermen. Es conveniente revisar el consumo del alimento mezclado, especialmente al comienzo puede presentarse una pequeña reducción, pero a partir el segundo día se normaliza.
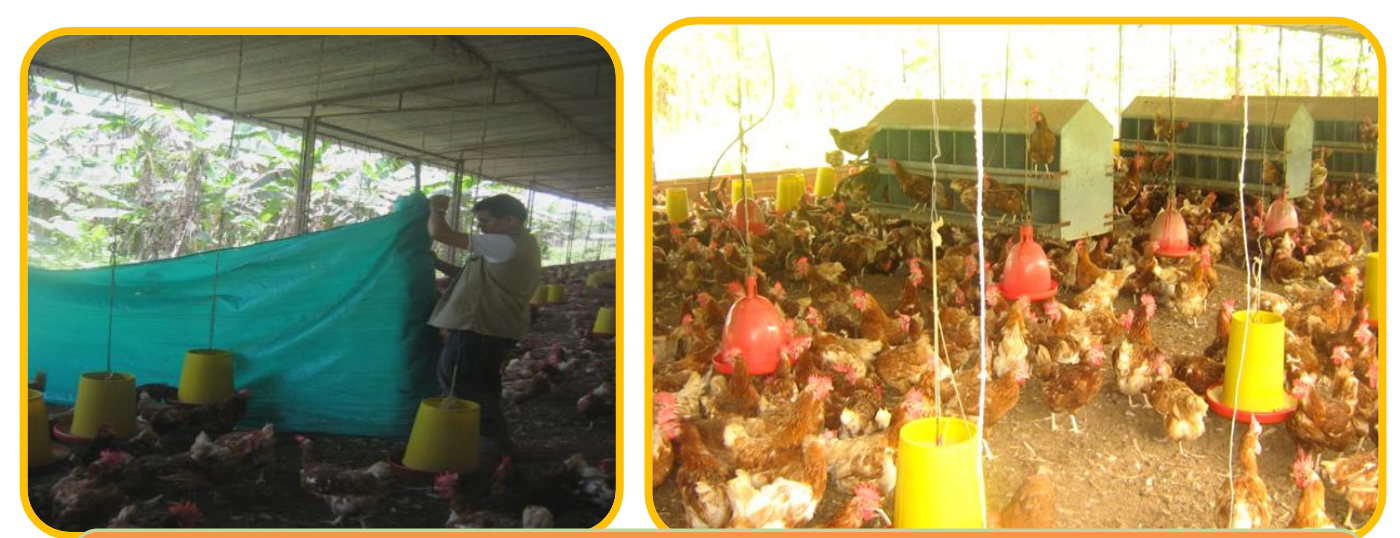

Figura 2. Selección de grupos de ponedoras para establecer el efecto de la harina de pimentón.

\section{RECOMENDACIONES}

Evitar que la luz natural o artificial llegue de manera directa al pimentón durante el troceado, secado, molido y mezclado, porque la luz degrada los carotenoides que la gallina necesita, esos pasos del procedimiento se deben trabajar en la penumbra, por eso sería conveniente trabajar en el atardecer o en el amanecer.

En el sitio donde se haga el secado, debe haber alguna corriente de aire o que se conecte un ventilador en la velocidad baja, puesto que las altas velocidades que pueden acelerar el proceso de secado no se recomiendan, porque pueden afectar la calidad.

\section{CONCLUSIONES}

La coloración de la yema es altamente sensible a la acción de los carotenoides que se encuentran en el pimentón, pues el incremento en el tono del color de la yema empieza a manifestarse antes de finalizar la primera semana de consumo 
de la mezcla de harina de pimentón con el alimento concentrado. En cuanto a la producción de huevo, el sub grupo E2 (con el 1\% de harina de pimentón), sin incrementar el consumo de alimento, presentó el mayor porcentaje de postura $(72,8 \%)$ y el mayor número de huevos semanales/ave $(4,93)$, con un incremento de 1,6 gr en el peso de los huevos generados y una pigmentación de la yema (9,6 110) con 6 puntos por encima del grupo testigo. Estas características lo determinan como el sub grupo más eficiente para los productores.

La combinación de estrategias formativas que se utilizaron en este trabajo, se complementaron entre sí, e incrementaron notoriamente las probabilidades de éxito en el proceso de capacitación informal dirigido hacia la población rural

Como resultado pedagógico se presenta la capacitación de veintinueve (29) personas entre operarios y directivos de AVIMETA. El procedimiento enseñado se continuó haciendo en la granja avícola "Agropecuaria río Ocoa", hasta que se eliminó el lote experimental, por vejez. En las siete (7) granjas restantes, el procedimiento no se ha iniciado porque en ellas no se tienen ponedoras mayores de cincuenta (50) semanas de edad.

\section{BIBLIOGRAFÍA}

1. Borrero, M. L. La alimentación de la gallina y el valor nutricional del huevo." Facultad de Ciencias. Universidad Javeriana. 2007

2. Correa F. Juan B., León D. Ruby E., Mosquera R. Claudia, Rodríguez M. Margarita M., Urrea G. F., Viáfara L. Carlos A. Acciones afirmativas y ciudadanía diferenciada étnico-racial negra, afrocolombiana, palenquera y razial. Universidad Nacional de Colombia. Facultad de Ciencias Humanas. Departamento de Trabajo Social, Centro de Estudios Sociales - CES, Grupo de investigación sobre igualdad racial, diversidad cultural, conflictos ambientales y racismos en las américas negras, Idcarán. 900 p. 2009

3. Cuevas M. R. El canario rojo. Ed Hispano Europea S.A, 2a Ed. Barcelona España. 128 p. 2009.

4. DANE. Encuesta Nacional Agropecuaria. Componente avícola. 2008

5. Ensminger M, E. Razas y reproducción de las aves de corral. Selección y descarte. Zootecnia general. Tercera edición. Editorial "El Ateneo" Buenos Aires. Argentina. 1980

6. Gobernación del Meta. Evaluaciones Agropecuarias. Informe de coyuntura. Evaluación pecuaria. Producción avícola. Unidad de Planeación y Desarrollo Rural. Secretaría de Agricultura, Ganadería y Desarrollo Rural. 2006 
7. FENAVI. Plan nacional de desarrollo 2006-2010 ¿Dónde está la avicultura?. No. 137.48 p. $4007 . \quad$ Disponible en: http://www.fenavi.org/images/stories/revistaavicultores/pdfs/revista-137.pdf

8. Jarén-Galán, M; Mínguez-Mosquera M. I. Quantitative and qualitative changes associated with heat treatments in the carotenoid content of paprika oleoresins. Journal Agriculture Food Chem. 1999

9. Jiménez O, C. Bioquímica de las partes del huevo. Revista Avicultura Andina. 2007.

10. Martínez M, A. Carotenoides. Facultad química farmacéutica. Universidad de Antioquia. 2003.

11. Meléndez M., Vicario M. I., Heredia J. F. Pigmentos carotenoides: consideraciones estructurales y fisicoquímicas. Archivos Latinoamericanos de Nutrición, 57 (2). 2007.

12. Mora S. J. D. La producción avícola en Colombia. Connotaciones. Facultad de Ciencias Agropecuarias. Universidad Nacional. 2007.

13. ICONTEC. NTC 1240. Clasificación de huevos frescos para consumo. 1997.

14. Ortiz R. J. Muda forzada en ponedoras: Como y cuando realizarla. Asociación de avicultores. Departamento técnico. Santa Cruz. Bolivia. 2004.

15. Ospina A. A. Características agroforestales de los huertos familiares. Documento interno. Cali, Colombia: Fundación Ecovivero, 29 p. 1995.

16. Owen R. Química de los Alimentos. Editorial Acribia S.A. Zaragoza. España. 2000.

17. Rivera G. O. Como llegó la gallina a Colombia. Industria Avícola Colombiana. 1996.

18. Rodríguez A. D. Carotenoides y preparación de alimentos: la retención de los Carotenoides Provitamina $\mathrm{A}$ en alimentos preparados, procesados y almacenados. Departamento de ciencias de alimentos. Facultad de Ing. de alimentos. Universidad Estadual de Campinas. SP Brasil. 100 p. 1999.

19. Rodríguez G. C. (Coord.) Más allá del desplazamiento: políticas, derechos y superación del desplazamiento forzado en Colombia / Universidad de los Andes, Facultad de Derecho, Ediciones Uniandes, Bogotá. 776 p. 2009.

20. SOLLA. Alimentos concentrados. Ponedoras Uno. 2009. Disponible en: http://www.solla.com/es/content/ponedorasi?linea=avicultura\&seccion=postura $\underline{\text { \&fase }=\text { produccion }}$ 